BULLETIN OF THE

AMERICAN MATHEMATICAL SOCIETY

Volume 80, Number 6, November 1974

\title{
ABRAHAM ADRIAN ALBERT \\ 1905-1972
}

BY NATHAN JACOBSON

Adrian Albert, one of the foremost algebraists of the world and President of the American Mathematical Society from 1965 to 1967, died on June 6, 1972. For almost a year before his death it had become apparent to his friends that his manner had altered from its customary vigor to one which was rather subdued. At first they attributed this to a letdown which might have resulted from Albert's having recently relinquished a very demanding administrative position (Dean of the Division of Physical Sciences at the University of Chicago) that he had held for a number of years. Eventually it became known that he was gravely ill of physical causes that had their origin in diabetes with which he had been afflicted for many years.

Albert was a first generation American and a second generation American mathematician following that of E. H. Moore, Oswald Veblen, L. E. Dickson and G. D. Birkhoff. His mother came to the United States from Kiev and his father came from England. ${ }^{1}$ The father had run away from his home in Vilna at the age of fourteen, and on arriving in England, he discarded his family name (which remains unknown) and took in its place the name Albert after the prince consort of Queen Victoria. Albert's father was something of a scholar, with a deep interest in English literature. He taught school for a while in England but after coming to the United States he became a salesman, a shopkeeper, and a manufacturer. Adrian was born when his father was fifty-five and his mother was thirty-five. It was a second marriage for both parents; his father's first wife had died in childbirth, and his mother was a widow with two children when she married his father. Adrian was the middle child of a set of three children which his parents had in common. He grew up in a family that was formally orthodox Jewish but not strongly religious. In common with most immigrant families of the period the family had a strong drive toward assimilation and a determination to make the most of the opportunities offered by a comparatively free society undergoing rapid economic expansion with no limits in sight.

Albert spent all of his school years in the Midwest and all but two of these in Chicago. He attended public schools at Chicago and at Iron

\footnotetext{
${ }^{1}$ We are indebted to Mrs. Frieda Albert for background material on Professor Albert's family. 
Mountain, Michigan, and entered the University of Chicago in 1922 where in rapid succession he earned a B.S. degree in 1926, an M.S. degree in 1927, and a Ph.D. in 1928. His advisor for his master's and his doctoral dissertations was Leonard Eugene Dickson. After his doctorate Albert spent a year at Princeton University as a National Research Council Fellow. He was attracted to Princeton by that great master of associative algebra theory, J. H. M. Wedderburn, who was then a professor at the university. Albert returned to Princeton in 1933, this time as one of the first group of temporary members of the Institute for Advanced Study.

Albert married Frieda Davis in 1927, and they had three children, Alan, Roy, and Nancy, one of whom, Roy, died of diabetes at the age of twenty-three.

Except for two years (1929-1931) as an Instructor at Columbia University and a number of visiting professorships (at Rio de Janeiro, Buenos Aires, University of Southern California, Yale, and the University of California at Los Angeles) all of Albert's academic career was spent at the University of Chicago. In 1960 he was named Eliakim Hastings Moore Distinguished Service Professor, and he served as Chairman of the Department of Mathematics for three years until he became Dean of the Division of Physical Sciences in 1962. He held this position until 1971 when he reached the mandatory retirement age of sixty-five for the deanship.

Of the mathematicians who influenced Albert most directly we should list the following: Dickson, who set the direction for almost all of Albert's research and whose books, Algebras and their Arithmetics (1923) and Algebren und ihre Zahlentheorie (1927), stimulated the great flowering of associative algebra theory of the 1930's; Wedderburn, whose elegant results and methods were an inspiration to Albert; Hermann Weyl, whose lectures on Lie groups and especially Lie algebras aroused Albert's interest in this subject-an interest which later broadened to encompass the whole range of nonassociative algebras; and above all, Solomon Lefschetz, who introduced Albert to the subject of Riemann matrices during his postdoctoral year (1928-1929) at Princeton.

Mrs. Albert tells the story of this introduction in a charming fashion. Filling in some mathematical details it runs somewhat as follows. Albert had given a lecture on his dissertation at the Princeton mathematics club. In the audience were Dieudonné, J. H. C. Whitehead and Lefschetz, who had worked on the problem of multiplication algebras of Riemann matrices. Lefschetz apparently sensed that here was a brilliant young algebraist whose interests and power made him ideally suited to attack this problem. After Albert's talk he described the problem to him. A lively discussion ensued, mostly in the course of wanderings through the streets 
of Princeton. This lasted for several hours, well past dinnertime, and Mrs. Albert had become quite concerned before Albert finally returned home, apparently in great excitement over his initiation into a fascinating area of classical mathematics which provided a strong motivation for the study of his chosen field of associative algebras.

Lefschetz was certainly right in his judgment. Albert took to the problem on Riemann matrices with great enthusiasm, and as the structure theory of associative algebras was revealed by Albert, Brauer, Hasse and Emmy Noether, Albert could push forward the theory of multiplication algebras of Riemann matrices until he achieved a complete solution of the central problem (which we shall discuss below). For this achievement Albert was awarded the Cole Prize in algebra in 1939.

This was a memorable year for Albert. Besides the Cole Prize award which he received that year, he was the Colloquium speaker of the Society for 1939. Moreover, he performed a feat, which we believe has never been matched, of having the book, Structure of Algebras, the subject of his lectures in print at the same time that the lectures were delivered.

Around 1942 Albert's research interests shifted from associative to nonassociative algebras. He wrote many important papers in this field (which we shall discuss below). In 1965 Albert returned to his first love, structure theory of associative algebras.

Besides his own important contributions to mathematics, Albert was instrumental in a number of ways in improving the status of the profession. He had a good deal to do with the establishment of government research grants for mathematics on more or less an equal footing with those in the other sciences. He was chairman of the Committee to Prepare a Budget for Mathematics for the National Science Foundation, 1950, and chairman of the Committee on a Survey of Training and Research Potential in the Mathematical Sciences, January 1955-June 1957 (which became known as "The Albert Committee"). He demonstrated that pure mathematicians could be useful in applied and directed research by acting as a consultant for a number of government sponsored research agencies. For a number of years he was associated with the Institute for Defense Analysis as a member of its Board of Trustees and for a year as Director of its Princeton group. He directed the research project SCAMP for several summers and organized and directed the project ALP (known as "Albert's little project"').

Albert was also a driving force in the creation of the summer research institutes which have become such an important part of the research activities of the Society, supported by the National Science Foundation. $\mathrm{He}$ was chairman of the committee which was responsible for the first 
one of these-on Lie groups and Lie algebras-held at Colby College in Maine in the summer of 1953.

Albert's role as a "statesman" for mathematics included membership on the Board of Trustees of the Institute for Advanced Study, chairman of the Consultative Committee of the Nice Congress, and Vice-President of the International Mathematical Union.

His influence in mathematics extended also through a large number of gifted students. One of the most distinguished of these, Dan Zelinsky, has written a warm appreciation of Albert as a mathematician and as a person [188].

Naturally many important honors came his way. He was elected to the National Academy of Sciences in 1943 and was awarded honorary doctorates from Notre Dame, Yeshiva University, and the University of Illinois. He was elected a corresponding member of the Brazilian Academy of Sciences, honorary member of the Argentine Academy of Sciences, and of the Mexican Mathematical Society. He thoroughly enjoyed these honors, but he derived almost as much pleasure from the honors bestowed on fellow algebraists and on his friends. Most of all he enjoyed seeking out a colleague to whom he could communicate his latest discovery, which excited him greatly.

Most of Albert's important discoveries fall neatly into three categories: I. Associative Algebras, II. Riemann matrices, III. Nonassociative algebras. We proceed to give an indication of these and of some interesting isolated results which we shall mention under IV. Miscellaneous.

I. Associative algebras. The Wedderburn structure theorems of 1907 on finite dimensional associative algebras over a field focused attention on the division algebras in this class. In 1906 Dickson had given a construction of a type of algebra called cyclic which included division algebras. These contain a maximal subfield 3 which is cyclic over the base field $\mathfrak{F}$, that is, they are Galois with Galois group $G=\langle s\rangle$, a cyclic group generated by a single element $s$. Moreover, the algebras are generated by 3 and an element $u$ for which one has the relations

$$
u z=s(z) u, \quad z \in 3, \quad u^{n}=\gamma,
$$

where $n$ is the order of $G$ and $\gamma$ is a nonzero element of $\mathfrak{F}$. The cyclic algebra, denoted as $(3, s, \gamma)$, constructed in this way has dimensionality $n^{2}$ over $\mathfrak{F}$. In 1914 Wedderburn [184] proved an important sufficient condition for $(3, s, \gamma)$ to be a division algebra. He showed that this is the case if no power of $\gamma, \gamma^{m}$ with $0<m<n$, is a norm $N_{3 / \Im}(z)$ of an element $z \in 3$. Using this criterion it is easy to construct division algebras of any dimension $n^{2}$.

In 1921 Wedderburn published some other important results on division 
algebras [185]. Noting that one may as well consider these as algebras over their centers and so assume that they are central in the sense that the center is the base field $\mathfrak{F}$, he showed that the dimensionality over this field is a square, $n^{2}$. More generally, if $\mathfrak{A}$ is central simple, by one of Wedderburn's structure theorems, $\mathfrak{A}$ is the algebra $M_{r}(\mathfrak{D})$ of $r \times r$ matrices with elements in a central division algebra $\mathfrak{D}$. Hence if the dimensionality of $\mathfrak{D}$ over $\mathfrak{F}$ is $d^{2}$, then that of $\mathfrak{A}$ over $\mathfrak{F}$ is $n^{2}$, where $n=d r$. Then $n$ is called the degree of the central simple algebra $\mathfrak{A}$ and $d$ is its index. In his 1921 paper, Wedderburn showed also that any maximal subfield 3 of a central division algebra $\mathfrak{D}$ is a splitting field, that is, the algebra $\mathfrak{D}^{3}=$ $3 \otimes_{\mathfrak{F}} \mathfrak{D}$ is the matrix algebra $M_{d}(3)$, and he proved that every central division algebra of degree three is cyclic. Wedderburn showed also that Dickson's cyclic algebras were special cases of a more 'general type of algebra which is now called an abelian crossed product. Here the cyclic field 3 is replaced by a Galois extension field of the base field with Galois group an abelian group.

Abelian crossed products were rediscovered by Cecioni [156], and these were further generalized by Dickson [158] and [159] to arbitrary crossed products based on any Galois extension field.

Much of Albert's early work was concerned with the study of finite dimensional central simple algebras. His first important result on these was the theorem, proved in his dissertation [9], that every central division algebra of degree four (dimension sixteen) is a crossed product. This was the next case to be considered after Wedderburn's theorem that in degree three these algebras are cyclic. Albert improved the result in [11] by showing that the degree four central division algebras are crossed products based on abelian extension fields whose Galois groups are direct products of two cyclic groups of order two, and he gave a simpler proof of this result in [41]. In both of these papers the algebras of characteristic two were excluded. In a subsequent paper [53] he was able to overcome the difficulties of the characteristic two case. Brauer was the first to show that the central division algebras of degree four, unlike those of degree three, need not be cyclic. He constructed an example of such an algebra which was a tensor product of two (generalized) quaternion algebras [153]. Subsequently, Albert [45] constructed one which is not such a product. This was significant in view of another important theorem, proved by Albert [39], stating that a central division algebra $\mathfrak{D}$ of degree four is a tensor product of quaternion algebras if and only if $\mathfrak{D} \otimes_{\mathfrak{F}} \mathfrak{D} \cong M_{4}(\mathfrak{F})$.

The main goal of the structure theory of algebras of the period 19291932 was the determination and classification of finite dimensional division algebras over the field $\boldsymbol{Q}$ of rational numbers, or equivalently, 
finite dimensional central division algebras over number fields. It was recognized quite early that this problem had two separate aspects: a purely algebraic one concerned with properties of algebras valid for all base fields, and an arithmetic one exploiting the arithmetic of number fields. Albert recognized the importance of the arithmetic method. However, he was handicapped in its use by the fact that he was unaware until rather late of the powerful results of algebraic number theory, notably, class field theory, which had been developed in Germany. He did make use of the arithmetic theory of quadratic forms to achieve definitive results on central division algebras of degree four over number fields and some important early results on the degree $2^{n}$ case. For example, he proved that the former are cyclic and are not tensor products of quaternion algebras, and he proved that the only central division algebras over number fields which possess involutions, that is, antiautomorphisms of period two, are the quaternion algebras. This last result was needed for his study of Riemann matrices which we shall discuss below.

Albert's main contributions were on the purely algebraic side. There is a substantial overlap between his results on central simple algebras and those of the German school of algebraists of the period of the early thirties, especially those of Richard Brauer and of Emmy Noether. Albert obtained independently all the algebraic results on splitting fields, extensions of isomorphisms and tensor products which were needed to obtain the fundamental theorems on division algebras over number fields. Of central importance for the algebraic theory is the group of classes of central simple algebras which was introduced by Brauer in 1929 [153]. We recall the definition. Two (finite dimensional) central simple algebras $\mathfrak{A}$ and $\mathfrak{B}$ over a field $\mathfrak{F}$ are said to be similar $(\sim)$ if there exist positive integers $m$ and $n$ such that the matrix algebras $M_{m}(\mathfrak{H})$ and $M_{n}(\mathfrak{B})$ are isomorphic. This is an equivalence relation. Denoting the similarity class of $\mathfrak{A}$ as $\{\mathfrak{A}\}$, one defines a product of such classes by $\{\mathfrak{Q}\}\{\mathfrak{B}\}=$ $\left\{\mathfrak{A} \otimes_{\mathfrak{F}} \mathfrak{B}\right\}$. This gives a commutative group $B(\mathfrak{F})$ called the Brauer group of the field $\mathfrak{F}$. The unit of the group is the set of matrix algebras $M_{n}(\mathfrak{\mho}), n=1,2, \cdots$, and the inverse of $\{\mathfrak{Q}\}$ is $\left\{\mathfrak{H}^{\mathrm{op}}\right\}$, where $\mathfrak{H}^{\mathrm{op}}$ is the opposite algebra of $\mathfrak{A}$. In a beautiful paper [33] published in 1931, Albert essentially rediscovered the Brauer group. In this paper he proved Brauer's main theorem that $B(\mathfrak{F})$ is a torsion group; more precisely, if $\mathfrak{A}$ has index $m$, that is, if the degree of the division algebra $\mathfrak{D}$ in $\{\mathfrak{U}\}$ is $m$, then $\{\mathfrak{Q}\}^{m}=1$. Moreover, if $e$ is the order of $\{\mathfrak{U}\}$ in $B(\mathfrak{\mho})$, then $e$ and $m$ have the same prime factors. The integer $e$ is called the exponent of $\mathfrak{A}$. Albert's proofs are based on Wedderburn's norm condition for cyclic algebras to be division algebras and theorems reducing considerations to the cyclic case; for example, if $\mathfrak{D}$ is a central division algebra of prime degree $p$, 
then there exists an extension field $\Omega$ of the base field of dimensionality prime to $p$ such that $\mathfrak{D}^{\mathfrak{R}}=\mathfrak{R} \otimes_{\mathfrak{F}} \mathfrak{D}$ is a cyclic division algebra over $\mathfrak{K}$. Another key tool in Albert's method was the following theorem which he called the index reduction factor theorem: Let $\mathfrak{D}$ be a central division algebra of degree $d$ and $\mathcal{R}$ an extension field of the base field $\mathfrak{F}$ with dimensionality $r$. Then $\mathfrak{D}^{\mathfrak{A}}=M_{q}(\mathfrak{E})$, where $\mathfrak{E}$ is a central division algebra over $\Re$ and $q$ is a divisor of $d$ and $r$. Albert's primary interest in the theorem that $\{\mathfrak{A}\}^{m}=1$ was its consequence that any central division algebra is a tensor product of division algebras of prime power degrees which are determined up to isomorphism. This reduced most questions on these algebras to the prime power degree case.

The high points of the structure theory of algebras of the 1930's were undoubtedly the theorem that every finite dimensional central division algebra over a number field is cyclic, and the classification of these algebras by a set of numerical invariants. The latter result amounts to the determination of the structure of the Brauer group for a number field. Besides the general theory of central simple algebras we have indicated, the proofs of these fundamental results required the structure theory of central simple algebras over $p$-adic fields due to Hasse, Hasse's norm theorem ("the Hasse principle"), and the Grünwald existence theorem for certain cyclic extensions of a number field. (Though it was discovered almost thirty years later by S. Wang [182] that Grünwald's formulation was incorrect, his error did not affect the theorem on algebras. See also Wang [183] and Hasse [161].) The first proof of the cyclic structure of central division algebras over number fields was given by Brauer, Hasse and Noether ([155], 1931). However, it seemed appropriate that Albert should share the honor of this achievement, and at Hasse's suggestion a joint paper ([42], 1932) was published by Albert and Hasse giving another proof of the theorem and the historical background of the problem.

The results which had been obtained up to this point suggested the following two problems: (I) Is every finite dimensional central division algebra a crossed product? (II) Is every one of prime degree cyclic? These are equivalent to the question of existence of a maximal Galois and maximal cyclic subfield, respectively, for these algebras. The results of Wedderburn and Albert imply that the answer to the second question is affirmative for the primes 2 and 3 and for the first for the degrees 2, 3, 4, 6 and 12 . Quite recently Amitsur showed that the answer to the first question is negative by showing that for any $n$ divisible by eight or by the square of an odd prime there exists a noncrossed product central division algebra of degree $n[\mathbf{1 5 0}]$. This leaves intact the second problem, and this is one on which Albert spent a good deal of effort. It is clear from the definition that if $\mathfrak{U}$ is cyclic of degree $n$, then $\mathfrak{A}$ contains an element $u$ satisfying an 
irreducible pure equation $x^{n}-\gamma=0, \gamma$ in the base field $\mathfrak{F}$. Does the converse hold? Albert showed this is the case if $n=p$, a prime ([59], 1934), and is not the case if $n=4$ ([72], 1938). For the prime case this reduces the problem (II) to what appears to be a more tractable one: Does every central division algebra $\mathfrak{A}$ of prime degree $p$ contain an element not in $\mathfrak{F}$ whose $p$ th power is in $\mathfrak{F}$ ? In 1938 Brauer showed that if $\mathfrak{A}$ is of degree 5 there exists a field $\Omega$ containing a tower of fields $\mathfrak{F} \subset \Omega_{1} \subset \Omega_{2} \subset \Omega$ such that the degree $\left[\mathfrak{R}_{1}: \mathfrak{F}\right]=2=\left[\mathfrak{R}_{2}: \mathfrak{R}_{1}\right]$ and $\left[\mathfrak{R}_{:} \mathfrak{R}_{2}\right]=3$, and $\mathfrak{H}^{\mathfrak{R}}$ is cyclic [154]. This led Albert to consider the following question: Suppose $\Omega$ is a quadratic extension of $\mathfrak{F}$ and $\mathfrak{A}^{\mathfrak{R}}$ is cyclic of prime degree. Then is $\mathfrak{A}$ cyclic? In four papers [142], [144], [147], [148] appearing between 1965 and 1970, including his retiring Presidential Address for the Society, Albert considered this problem for algebras of characteristic $p$ and degree $p$. In spite of many ingenious arguments and partial results, he was unable to completely settle this question.

A beautiful chapter in the structure theory of central simple algebras is the theory of $p$-algebras which Albert developed in three papers [66], [67], [69] appearing in 1936 and 1937 (cf. also [181]). These are the central simple algebras of characteristic $p$ whose division algebras $\mathfrak{D}$ in the Wedderburn theorem $\left(\cong M_{n}(\mathfrak{D})\right)$ have degree a power of $p$. The main results Albert proved about $p$-algebras are that any such algebra $\mathfrak{A}$ is cyclically representable, that is, there exists an $n$ such that $M_{n}(\mathfrak{U})$ is a cyclic algebra, and the exponent of $\mathfrak{A}$ is the minimum of the exponents of purely inseparable splitting fields for the algebra.

A generalization of cyclic algebras in which the cyclic maximal subfield 3 is replaced by a separable commutative subalgebra on which a cyclic group $G$ acts in such a way that there are no proper subalgebras stabilized by $G$ was considered by Albert in [75] following earlier work by Teichmüller in [180]. Such generalized cyclic algebras arise naturally from cyclic ones when one extends the base field or forms the tensor powers of a cyclic algebra.

Most of the important results on associative algebras which Albert obtained prior to 1939 can be found in an improved form in his AMS Colloquium book, Structure of Algebras. This extemely readable and beautifully organized book can still be recommended to a beginning student with a serious interest in structure theory and is an indispensable reference book for certain aspects of the theory, particularly the theory of $p$-algebras, and of algebras with involution.

II. Riemann matrices and associative algebras with involution. The theory of multiplications of Riemann matrices has its origin in algebraic geometry. On a Riemann surface of an algebraic curve of genus $p$, one 
chooses $p$ linearly independent integrals of the first kind each with $2 p$ periods $\omega_{j v}, 1 \leqq j \leqq p, 1 \leqq v \leqq 2 p$. The $p \times 2 p$ matrix $\omega=\left(\omega_{j v}\right)$ of complex elements satisfies the Riemann relations: there exists a $2 p \times 2 p$ nonsingular skew symmetric matrix $C$ of rational elements such that $\omega C^{t} \omega=0\left({ }^{t} \omega\right.$ the transpose of $\omega$ ) and $\sqrt{-1} \omega C^{t} \bar{\omega}$ is positive definite hermitian. In the theory of so-called singular correspondences on the Riemann surface, one is led to consider the multiplications of $\omega$. These are the $2 p \times 2 p$ rational matrices $A$ for which there exists a $p \times p$ complex matrix $\alpha$ such that $\alpha \omega=\omega A$. The set of these $A$ 's is a finite dimensional algebra of matrices over $\boldsymbol{Q}$, the algebra of multiplications of $\omega$.

Alternatively, the matrices $\omega$ and their multiplications arise in the theory of abelian functions, defined to be meromorphic functions of $p$ complex variables having a lattice of periods in $\boldsymbol{C}^{p}$.

There is another, formally simpler, formulation of Riemann matrices (the foregoing $\omega$ ) and their multiplications due to Weyl [186] which was suggested by geometric considerations. From the purely formal point of view one obtains the passage from the classical formulation to Weyl's by introducing the $2 p \times 2 p$ matrix

$$
W=\left(\begin{array}{l}
\omega \\
\bar{\omega}
\end{array}\right) \quad \text { and } \quad L=\left(\begin{array}{ccc}
-\sqrt{-1} & 1_{p} & 0 \\
0 & \sqrt{-1} 1_{p}
\end{array}\right) .
$$

Put $R=W^{-1} L W$. Then it can be shown that the matrix $R$ has the following properties: (1) $R$ is real, that is $R \in M_{2 p}(R)$; (2) $R^{2}=-1_{2 p}$; (3) $S=C R$ is positive definite symmetric. Following Weyl, one calls a matrix $R \in M_{n}(R)$ (here $n=2 p$ ) a Riemann matrix if $R^{2}=-1_{n}$ and there exists a skew symmetric matrix $C \in M_{n}(Q)$ such that $S=C R$ is positive definite symmetric. The matrix $C$, which is necessarily nonsingular, is called a principal matrix of $R$. The passage from Weyl's $R$ to the classical $\omega$ can be reversed. In Weyl's formulation the multiplications appear as the matrices $A \in M_{n}(Q)$ commuting with $R$. The set $\mathfrak{A}$ of these multiplications is a finite dimensional algebra over $\boldsymbol{Q}$ called the multiplication algebra of the Riemann matrix $R$. Weyl observed that for most considerations the condition $R^{2}=-1$ $\left(=-1_{n}\right)$ plays no role. Dropping this, one obtains generalized Riemann matrices. Subsequently Albert [2], [65] considered further generalizations (including even a characteristic $p \neq 0$ situation!). For the sake of simplicity we shall stick to the case of Riemann matrices in Weyl's formulation.

The important early work on multiplication algebras is due to Poincaré, Scorza, Lefschetz and Rosati. Poincaré achieved a reduction to so-called pure Riemann matrices for which the multiplication algebras are division algebras. Lefschetz considered the situation in which the multiplication algebras are commutative. Rosati observed the important fact that if $A$ is in the multiplication algebra $\mathfrak{A}$ of a Riemann matrix $R$ and $C$ is a 
principal matrix, then $A^{*}=C^{-1}{ }^{t} A C \in \mathfrak{A}$. The map $A \rightarrow A^{*}$ is an involution (antiautomorphism of period two) in $\mathfrak{A}$. Rosati showed also that if $A$ is symmetric under this involution $\left(A^{*}=A\right)$ then its characteristic roots are real, and if $A^{*}=-A$ then its characteristic roots are pure imaginaries [176].

The central problem on multiplication algebras of Riemann matrices is to determine necessary and sufficient conditions that a division algebra over $\boldsymbol{Q}$ be the multiplication algebra of a Riemann matrix. For a proof of sufficiency, one requires a construction of a Riemann matrix whose multiplication algebra is a given algebra $\mathfrak{A}$ satisfying the conditions.

Albert's work on Riemann matrices went hand in hand with the development of the theory of division algebras. It culminated in the complete solution of the principal problem, which he published in three papers appearing in the Annals of Mathematics in 1934 and 1935 ([51], [57], and [63]). To achieve this required the development $\mathrm{ab}$ initio of the basic theory of simple algebras with involution. Albert presented improved versions of this theory in [65] and in his Structure of Algebras. We shall indicate first his results on algebras with involution.

We assume throughout that $\mathfrak{A}$ is finite dimensional simple over a field $\mathfrak{F}$. If $\mathfrak{A}$ has an involution $J\left(J: a \rightarrow a^{*}\right.$ such that $(a+b)^{*}=a^{*}+b^{*},(\alpha a)^{*}=\alpha a^{*}$ for $\left.\alpha \in \mathfrak{F},(a b)^{*}=b^{*} a^{*}\right)$, then the center $\mathfrak{C}$ of $\mathfrak{A}$ is stabilized by $J$ and the restriction of $J$ to $\mathfrak{C}$ is either the identity map or an automorphism of period two. Accordingly, the involution is of first kind or second kind. Albert showed that $\mathfrak{A}$ has an involution if and only if for any $m=1$, $2, \cdots$, the matrix algebra $M_{m}(\mathfrak{U})$ has an involution having the same effect on the center (which can be identified with the center of the matrix algebra). He showed also that if $\mathfrak{A}$ has an involution $J$ and $x$ is an element of $\mathfrak{A}$ whose minimum polynomial over the center $\mathfrak{C}$ is irreducible and has coefficients that are $J$-symmetric, then $\mathfrak{A}$ has an involution $T$ leaving $x$ fixed and having the same effect on $\mathfrak{C}$ as $J$. Assuming $\mathfrak{A}$ is of dimension $n^{2}$ over its center $\mathfrak{C}$ and contains a subfield of the form $\mathfrak{X} \otimes_{\mathfrak{F}} \mathfrak{C}$, where $\mathfrak{X}$ is $n$-dimensional Galois over $\mathfrak{F}$, and $\mathfrak{C}$ is either $\mathfrak{F}$ or a separable quadratic extension of $\mathfrak{F}$, he gave a necessary and sufficient condition in terms of a factor set for $\mathfrak{A}$ to have an involution. This was used to give constructions which in principle yield all simple algebras with involution. Albert also used these results to prove that a central simple algebra has an involution if and only if it has exponent one or two in the Brauer group. One can combine this with one of the results of $I$ to conclude that a central division algebra of degree four has an involution if and only if it is a tensor product of quaternion algebras. It seems unlikely that this is true for degree greater than four but we believe that this remains an open question.

Albert proved that if a division algebra over a number field has an involution of first kind, then the algebra is a quaternion algebra over its 
center. Moreover, he determined the division algebras over number fields having involutions of second kind. He showed that any such algebra is cyclic $(\mathfrak{Z}, s, \gamma)$ over its center $\mathfrak{C}$, where the cyclic field $\mathbb{Z}$ over $\mathfrak{C}$ has the form $3_{0} \otimes_{\mathfrak{C}_{0}} \mathfrak{C}, \mathfrak{C}_{0}$ the subfield of symmetric elements of $\mathfrak{C}, 3_{0}$ cyclic over $\mathfrak{C}_{0}$, and $\mathfrak{C}$ separable quadratic over $\mathfrak{C}_{0}$. Moreover, $\gamma \in \mathfrak{C}$, and if $\bar{\gamma}$ is its conjugate in $\mathfrak{C}$ over $\mathfrak{C}_{0}$, then $\gamma \bar{\gamma}$ is a norm of an element of $3_{0}$. Conversely any division algebra having the indicated cyclic structure does have an involution of second kind. In a later paper [137], Albert obtained a similar result for division algebras of degree three (over their centers) for arbitrary base fields.

These results, especially those on division algebras with involutions over number fields, provided the machinery for the solution of the problem of multiplication algebras for Riemann matrices. Using Rosati's theorem, one sees that the center $\mathfrak{C}$ of such an algebra is totally real if the (Rosati) involution is of first kind and is a pure imaginary quadratic extension of a totally real field if the involution is of second kind. Besides these conditions on $\mathfrak{C}$, there are supplementary conditions on the quaternion division algebras and the cyclic algebras, which occurred in our description of the division algebras with involution over number fields, that must be fulfilled for these to be multiplication algebras of Riemann matrices. In Albert's proof of the sufficiency of the conditions he had derived, he made use of the Hilbert irreducibility theorem for number fields.

In an exposition [178] of the theory of Riemann matrices, C. L. Siegel made some notable improvements on Albert's results. We should mention also that Weyl in [187] gave an alternative treatment of the subject based on Brauer factor sets.

III. Nonassociative algebras. From about 1942 to 1965, when he returned to the problem of existence of noncyclic associative division algebras of prime degree, most of Albert's research was in the area of nonassociative algebra: structure theory of nonassociative algebras, quasigroups, nonassociative division rings, and nondesarguesian projective planes. In our account of his contributions to this rather broad field of mathematics, we shall be selective, picking out what we consider his most important work-judged from the criterion of general mathematical interest. From this point of view, Albert's discoveries on Jordan algebras are undoubtedly his most important ones in nonassociative algebra, and these are perhaps on a par with his work on associative algebras and Riemann matrices. We shall begin our account with this work, and we shall first sketch the story of Jordan algebras before Albert took them up as a subject of intensive study.

The study of the class of algebras which now bear his name was initiated in 1932 by the physicist, P. Jordan. His declared objective was to 
achieve a better formalism for quantum mechanics than one based on selfadjoint operators in Hilbert space. Observing that the set of these operators is a vector space over $\boldsymbol{R}$ which is closed under the product $A \cdot B=\frac{1}{2}(A B+B A)$, where $A B$ is the usual associative product, and that this symmetrized product is commutative and satisfies the identity $\left(A^{2} \cdot B\right)$. $A=A^{2} \cdot(B \cdot A)$, he proposed to consider algebras in which the product composition satisfies these two conditions. He enlisted the help of von Neumann and Wigner in his study, and as a result of their collaboration, there appeared, in 1934, a paper entitled On an algebraic generalization of the quantum mechanical formalism which was a gem in the structure theory of algebras. In this paper [168], Jordan, von Neumann and Wigner obtained a complete determination of the finite dimensional (nonassociative) algebras over $\boldsymbol{R}$ satisfying the following conditions: I. Formal reality in the sense of Artin-Schreier, that is, the requirement that the only relations of the form $\sum a_{i}^{2}=0$ in the algebras are the trivial ones in which every $a_{i}=0$. II. Commutativity of the product $(a b=b a)$ and the identity $\left(a^{2} b\right) a=a^{2}(b a)$.

They showed that the algebras satisfying these conditions are direct sums of ideals that are simple algebras, and they determined the simple ones as belonging to one of the following classes:

(1) The vector space over $\boldsymbol{R}$ of $n \times n$ hermitian matrices with entries in $\boldsymbol{R}, \boldsymbol{C}$, or Hamilton's quaternion algebra $\boldsymbol{H}$, endowed with the algebra structure in which the product is $a \cdot b=\frac{1}{2}(a b+b a)$ in terms of the usual matrix product $a b$.

(2) The algebras over $\boldsymbol{R}$ with bases $\left(1, e_{1}, e_{2}, \cdots, e_{n}\right)$ and multiplication defined by the table $e_{i} e_{j}=\delta_{i j} 1,1$ the unit.

(3) The algebra $M_{3}^{8}$ of $3 \times 3$ hermitian matrices with entries in the algebra $\boldsymbol{O}$ of Cayley numbers, endowed with the product $a \cdot b=\frac{1}{2}(a b+b a)$, where, as before, $a b$ is the usual matrix product.

Conversely, the algebras listed satisfy the above conditions.

Now one defines a Jordan algebra over a field $\mathfrak{F}$ of characteristic $\neq 2$ as an algebra in which the product $a b$ satisfies

$$
a b=b a, \quad\left(a^{2} b\right) a=a^{2}(b a) .
$$

Among these are included the special Jordan algebras which are isomorphic to subspaces of an associative algebra closed under the Jordan product $a \cdot b=\frac{1}{2}(a b+b a)$ and regarded as algebras relative to this product. Evidently, the algebras in Jordan, von Neumann, Wigner's class (1) are special. It is easy to see, using Clifford algebras, that this is the case also for algebras of their class (2). They conjectured that $M_{3}^{8}$ is not special, and they proposed the proof of this as a problem to Albert, who showed that this is indeed the case: $M_{3}^{8}$ is an exceptional (=nonspecial) Jordan algebra [52]. 
After this brief encounter with the Jordan theory, a number of years elapsed before Albert returned to the subject. In a series of three papers [94], [97], [105] appearing in 1946, 1947 and 1950, Albert developed the basic structure theory of finite dimensional Jordan algebras over a field of characteristic not two. Since it is interesting to observe how the subject evolved in Albert's hands, we shall give a brief indication of the contents of each of these papers.

In the first one he considered Jordan algebras defined concretely as Jordan algebras of linear transformations of a finite dimensional vector space, that is, subspaces of End $\mathfrak{B}$ closed under the Jordan product $A \cdot B$. He proved analogues for these algebras of Lie's and Engel's theorems on Lie algebras. Assuming the base field is of characteristic 0 (so trace arguments can be used) he showed that if the algebras contain no nil ideals they are direct sums of simple algebras. Moreover, he determined the simple algebras over an algebraically closed field of characteristic 0 . This determination is quite similar to that of Jordan, von Neumann and Wigner's of simple formally real Jordan algebras: Class (2) is unchanged, and the modification required in the definition of class (1) is that $\boldsymbol{R}, \boldsymbol{C}$ and $\boldsymbol{H}$ be replaced by the split composition algebras of dimensions 1,2 and 4 over the given base fields. (These are the algebras which occur in Hurwitz's problem on quadratic forms permitting composition.) Naturally there is no class (3) since the algebras under consideration are special by definition. Actually Albert determined a more general class of so-called reduced algebras over an arbitrary field of characteristic 0 . However, the result he obtained in the algebraically closed case is adequate to permit the determination of all the special simple algebras over an arbitrary field using the method of descent. This was done by Kalisch [169] and F. D. Jacobson and N. Jacobson [163].

In his second paper, Albert dealt with abstract finite dimensional Jordan algebras over any field of characteristic not two. He showed that nil algebras of this type are nilpotent in the sense that there exists an integer $r$ such that the product of any $r$ elements of the algebra in any association is 0 . He proved that if the characteristic is 0 and $\mathfrak{A}$ has no nil ideals $\neq 0$, then $\mathfrak{A}$ is a direct sum of simple algebras, and he determined the simple ones over an algebraically closed field of characteristic 0 . Here, one does have exceptional algebras, and the only simple one over an algebraically closed field of characteristic 0 is the analogue of $M_{3}^{8}$ in which the classical Cayley algebra is replaced by a split Cayley algebra. We shall refer to this as the split exceptional simple Jordan algebra.

In his third paper, Albert extended these results except for a small gap (which was filled by Jacobson in [165]) to the characteristic $p \neq 2$ case. 
A particularly interesting class of Jordan algebras is that of the finite dimensional exceptional central simple Jordan algebras. If $\mathfrak{F}$ is the base field and $\overline{\mathfrak{F}}$ is its algebraic closure, then $\mathfrak{A}$ is in this class if and only if the extension algebra $\mathfrak{A}^{\widetilde{F}}$ is the split exceptional simple Jordan algebra previously defined. The theory of these exceptional algebras is intertwined with a number of other "exceptional" phenomena, notably, exceptional Lie groups and exceptional geometries (e.g. Cayley projective planes). Albert made a number of important contributions to this theory, sometimes in collaboration with others. We have already noted that his first paper on Jordan algebras established the exceptional character of $M_{3}^{8}$. In 1959 Albert and Paige, in a joint paper [129], proved a much stronger result: $M_{3}^{8}$ is not a homomorphic image of any special Jordan algebra. As a consequence of this and a result of Cohn's [157], one can conclude that the free Jordan algebra with three generators is not special.

One can distinguish two types of exceptional simple Jordan algebras: the reduced ones and the division algebras. The first contains idempotents $\neq 0,1$, and in the second the subalgebras generated by single elements and 1 are associative fields. It can be shown that the reduced ones have the form $\mathfrak{H}\left(\boldsymbol{O}_{3}, \gamma\right)$ the algebra of $3 \times 3$ matrices $A$ with entries in some (generalized) Cayley algebra $\boldsymbol{O}$, which are $\gamma$-hermitian in the sense that $\gamma^{-1}{ }^{t} \bar{A} \gamma=A$, where $\gamma$ is a 3-rowed diagonal matrix with entries in the base field, and $\bar{A}$ is obtained by replacing each Cayley number entry $a_{i j}$ by its conjugate $\tilde{a}_{i j}$. The problem of determining conditions for the isomorphism of two such algebras was studied by Albert and Jacobson [122]. It was shown in this paper that isomorphism of the Jordan algebras implied isomorphism of the Cayley algebras occurring in their definitions, and they obtained some rather complicated supplementary conditions for isomorphism. These sufficed to give a complete classification of reduced exceptional simple Jordan algebras over number fields. (See also [179] and [175].)

The first construction of exceptional Jordan division algebras is due to Albert [126], [141]. He showed also that no such algebras exist over number fields. On the other hand, if $\mathfrak{F}$ is any field over which there exist central associative division algebras of degree three (e.g. a number field), then there exist exceptional Jordan division algebras over the field $\mathfrak{F}(t)$ obtained by adjoining an indeterminate $t$ to $\mathfrak{F}$. Albert used a method of descent in his study of exceptional Jordan division algebras. Subsequently considerably simpler "rational" constructions were given by Tits [166, p. 412].

From the abstract point of view, a very natural class of algebras (or rings) is the class satisfying the power associativity condition: subalgebras (or subrings) generated by single elements are associative. This includes Jordan algebras, alternative algebras (defined by the identities 
$a^{2} b=a(a b)$ and $\left.b a^{2}=(b a) a\right)$, associative algebras, and a number of other interesting types of algebras.

Albert initiated the study of power associative rings (without finiteness conditions) in a paper [98] published in 1948. The conditions of power associativity are that for any $a$ one has the power formula

$$
a^{m} a^{n}=a^{m+n}, \quad m, n=1,2,3, \cdots,
$$

where $a^{m}$ is defined inductively by $a^{1}=a, a^{k}=a^{k-1} a$. In [98] Albert showed that if the additive group of a nonassociative ring $\mathfrak{A}$ has no torsion, then $\mathfrak{A}$ is power associative if and only if it satisfies the two identities $a a^{2}=a^{3}$ and $\left(a^{2}\right)^{2}=a^{4}$. These results were obtained by some clever inductive arguments based on linearizations of the assumed identities. These linearizations and commutativity yield also the crucial result that in a commutative power associative ring the map $e_{R}: x \rightarrow x e$, determined by an idempotent $e$, satisfies the quadratic equation $\left(2 e_{R}-1\right)\left(e_{R}-1\right) e_{R}=0$. If one assumes that the additive group admits the operator $\frac{1}{2}$, then one obtains the Peirce decomposition relative to

$$
e: \mathfrak{A}=\mathfrak{A}_{0}(e) \oplus \mathfrak{U}_{1 / 2}(e) \oplus \mathfrak{A}_{1}(e) \quad \text { where } \mathfrak{A}_{\imath}(e)=\left\{x_{\imath} \mid x_{\imath} e=i x_{i}\right\} .
$$

One also has extensions of this to Peirce decompositions relative to orthogonal idempotents. In Albert's hands, these Peirce decompositions became powerful tools for investigating power associative rings. He obtained a number of striking results by this method. We mention two:

Let $\mathfrak{A}$ be a simple commutative power associative ring whose additive group contains no elements of orders 2,3 or 5 and admits the operator $\frac{1}{2}$. Suppose $\mathfrak{A}$ contains two nonzero orthogonal idempotents $e$ and $f$ such that $e+f$ is not a unit. Then $\mathfrak{A}$ satisfies the Jordan identity $\left(a^{2} b\right) a=$ $a^{2}(b a)[\mathbf{1 0 5}]$.

Any simple alternative ring containing an idempotent $e \neq 0,1$ is either associative or a Cayley algebra over its center [109].

The ultimate result on simple alternative rings is due to Kleinfeld [170], [171]. This states that all simple alternative rings are either associative or Cayley algebras. Albert's theorem was used as a step in the first proof of Kleinfeld's theorem.

In [108] and [126] Albert proved a generalization for power associative rings of Wedderburn's celebrated theorem on the commutativity of finite associative division rings. Call an algebra over a field strictly power associative if all the algebras obtained by extending the base field are power associative. Also one defines a (nonassociative) division ring by the property that the left and right multiplications $x \rightarrow a x$ and $x \rightarrow x a$ are bijective for any $a \neq 0$ in the ring. Then Albert proved that any finite strictly power associative division algebra of characteristic $\neq 2$ is associative and commutative. Albert based his proof on the determination of the 
simple Jordan algebras over an arbitary field due to F. D. Jacobson and N. Jacobson [163] and a result of his own on exceptional Jordan algebras [126]. (Later McCrimmon gave alternative proofs which are independent of the structure theory [173], [174].) A number of constructions of nonassociative and noncommutative division rings are due to Albert [135], [108], [132]. These yield examples of nondesarguesian projective planes including some finite ones.

Albert had a hand in the discovery of several new classes of simple Lie algebras of prime characteristic (see [160], [113] and [151]). Recently these results have taken on added luster because of the discovery by Kostrikin and Shafarevitch [172] that these Lie algebras can be regarded as characteristic $p$ versions of infinite dimensional Lie algebras which had occurred in Eli Cartan's work on contact transformations.

Albert and his students and followers also studied a number of other classes of nonassociative algebras defined by identities. Until now the results which have been obtained on these appear to be of interest only to specialists in the field. We shall therefore refrain from giving any indication of these results. Albert wrote several papers on general nonassociative theory. In one of these [84] he gave a definition of a radical for any finite dimensional nonassociative algebra. Since the theory of the radical is quite interesting and deserves to be better known than it is at present, we take this opportunity to sketch what we believe is an improved version of this theory.

Let $\mathfrak{A}$ be a finite dimensional nonassociative algebra over a field. Then $\mathfrak{A}$ is called simple if $\mathfrak{A}^{2} \neq 0$ and $\mathfrak{A}$ has no ideals $\neq 0, \mathfrak{A}$. $\mathfrak{A}$ is semisimple if it is a direct sum of ideals which are simple algebras. Following the pattern of associative ring theory, it is natural to define the radical rad $\mathfrak{A}$ to be the intersection of the set of ideals $\mathfrak{B}$ of $\mathfrak{A}$ such that $\mathfrak{A} / \mathfrak{B}$ is simple. This definition implies that if no $\mathfrak{B}$ 's, such that $\mathfrak{U} / \mathfrak{B}$ is simple, exist then $\mathfrak{A}=$ $\operatorname{rad} \mathfrak{A}$. In any case $\mathfrak{A} / \mathrm{rad} \mathfrak{A}$ is semisimple or 0 , and $\operatorname{rad} \mathfrak{A}$ is contained in every ideal $\mathfrak{D}$ of $\mathfrak{A}$ such that $\mathfrak{U} / \mathfrak{D}$ is semisimple (see, for example, Jacobson, Structure of Rings, p. 41). This implies that $A \neq 0$ is semisimple if and only if $\operatorname{rad} \mathfrak{A}=0$.

One obtains important information on an algebra $\mathfrak{A}$ in looking at its multiplication algebra $M(\mathfrak{H})$. This is the subalgebra of the associative algebra End $\mathfrak{A}$ of linear transformations in $\mathfrak{A}$ generated by 1 and the left and right multiplications $\left(a_{L}: x \rightarrow a x, a_{R}: x \rightarrow x a\right)$ of $\mathfrak{A}$. The centralizer of $M(\mathfrak{l})$ in End $\mathfrak{A}$ is called the centroid $C(\mathfrak{H})$ of $\mathfrak{A}$. The study of $M(\mathfrak{H})$ and $C(\mathfrak{R})$ was initiated by Jacobson [164] (see also Jacobson, Lie Algebras, pp. 290-295). Albert's results on rad $\mathfrak{A}$, as we shall show, amount to a formula for $\operatorname{rad} \mathfrak{A}$ in terms of $\operatorname{rad} M(\mathfrak{H})$. We shall call $\mathfrak{A}$ reductive if $\mathfrak{A}$ is a direct sum of ideals which are simple algebras and the ideal 
$\mathcal{Z}=\{z \mid \mathfrak{A} z=0=z \mathfrak{U}\}$. The elements of 3 are called absolute zero divisors. One can show that $\mathfrak{A} \neq 0$ is reductive if and only if $M(\mathfrak{U})$ is semisimple. Hence $\mathfrak{A}$ is semisimple if and only if $M(\mathfrak{A})$ is semisimple and 0 is the only absolute zero divisor in $\mathfrak{A}$. Now let $\mathfrak{N}$ be the radical of $M(\mathfrak{U})$ and $\mathfrak{R}$ the ideal in $\mathfrak{A}$ such that $\mathfrak{R} / \mathfrak{N A}$ is the ideal of absolute zero divisors of $\mathfrak{A} / \mathfrak{N A}$. Then $\mathfrak{R}=\operatorname{rad} \mathfrak{A}$. Albert's order of ideas in his paper on the radical is the reverse of what we have indicated; namely, he uses the ideal $\Re$ as his definition of the radical, then proves it has the two basic properties that $\mathfrak{A} / \mathfrak{R}$ is semisimple and $\mathfrak{R}$ is contained in every ideal $\mathfrak{D}$ such that $\mathfrak{X} / \mathfrak{D}$ is semisimple.

For certain important classes of algebras (e.g. associative, alternative, Jordan), rad $\mathfrak{A}$ coincides with the maximal nil ideal. For Lie algebras of characteristic $0, \operatorname{rad} \mathfrak{A}$ is the maximal solvable ideal. On the other hand, Albert has given an example of an algebra in which rad $\mathfrak{A}$ is an associative field. We could not resist recording here a result on the radical which we have known for some time. This is a generalization of a well-known theorem of Hochschild's [162] on derivations of associative and Lie algebras.

THEOREM. If $\mathfrak{A}$ is a finite dimensional nonassociative algebra over a field of characteristic 0 then any derivation of $\mathfrak{A}$ stabilizes rad $\mathfrak{A}$.

This can be proved by using the fact that the Lie algebra Der $\mathfrak{A}$ of derivations is the Lie algebra of algebraic groups of automorphisms of $\mathfrak{A}$. A more direct proof, which is applicable also in some situations in characteristic $p \neq 0$, can be based on Albert's definition of rad $\mathfrak{A}$. We observe first that if $D$ is a derivation in $\mathfrak{A}$, then

$$
\left[D, a_{L}\right] \equiv D a_{L}-a_{L} D=(D a)_{L} \quad \text { and } \quad\left[D, a_{R}\right]=(D a)_{R} \text {. }
$$

Hence $m \rightarrow[D, m]$ is a derivation in $M(\mathfrak{A})$ which we denote as $\tilde{D}$. If $m \in$ $M(\mathfrak{A})$ and $a \in \mathfrak{A}$, then $D(m a)=(\widetilde{D} m) a+m(D a)$. It is easily seen that $D$ stabilizes rad $\mathfrak{A}$ if $\tilde{D}$ stabilizes $\operatorname{rad} M(\mathfrak{U})$. Our theorem then follows from Hochschild's theorem on associative algebras.

In a paper [82] which appeared in 1942, Albert introduced a concept of isotopy for nonassociative algebras. Let $\mathfrak{A}$ and $\mathfrak{B}$ be nonassociative algebras. Then $\mathfrak{A}$ and $\mathfrak{B}$ are called isotopes if there exist bijective linear maps $P$ and $Q$ from $\mathfrak{B}$ to $\mathfrak{A}$ and a bijective linear map $C$ from $\mathfrak{A}$ to $\mathfrak{B}$ such that for $x, y \in \mathfrak{B}$ we have

$$
x y=C((P x)(Q y)) .
$$

If $P=Q$ and $C=P^{-1}$, we have $P(x y)=(P x)(P y)$, so $P$ is an isomorphism. Isotopy is an equivalence relation. If $\mathfrak{B}$ and $\mathfrak{A}$ are identical as sets, and $C=1$, we call $\mathfrak{B}$ a principal isotope of $\mathfrak{A}$. Define a new multiplication on $\mathfrak{A}$ by $u \circ v=(P C u)(Q C v)$. This, along with the given vector space structure, 
gives a new algebra which is a principal isotope of $\mathfrak{A}$, and since

$$
x y=C((P x)(Q y))=C\left(\left(P C C^{-1} x\right)\left(Q C C^{-1} y\right)\right)=C\left(C^{-1} x \circ C^{-1} y\right),
$$

$\mathfrak{B}$ is isomorphic to the principal isotope defined by $\circ$. This reduces the consideration to that of principal isotopes. Albert defined isotopy also for quasigroups [87], and he proved a number of interesting results on isotopy of algebras and of quasigroups. While these have not played an important role in structure theory, the concept of isotopy has some importance in nondesarguesian geometry (see [134]).

IV. Miscellaneous. Albert wrote a number of papers [50], [54], [57], [58], [64] on the structure of field extensions. He was particularly interested in explicit constructions of cyclic field extensions since these played an important role in his investigations of the structure of division algebras. Albert's results on cyclic extensions are presented in a connected fashion in Chapter IX of his algebra text Modern Higher Algebra. There are numerous references to these results in Structure of Algebras. For the case of degree $p^{e}$ and characteristic $p$, one has an alternative method due to Witt, based on Witt vectors, which provides a better survey of cyclic and abelian extensions (see for example, Jacobson's Lectures in Abstract Algebra, vol. III, pp. 124-140). On the other hand, Albert's results on cyclic fields of degree $p^{e}$ and characteristic $\neq p$ seem not to have been improved upon until now.

Albert was fascinated by the problem of minimum number of generators for algebraic structures. He proved [92] that any separable associative algebra is generated by two elements and, with John Thompson [130], proved that the projective unimodular group over a finite field is generated by two elements, one of which has order two.

In a joint paper with Muckenhoupt [120], he proved that for any field $\mathfrak{F}$, any matrix of trace 0 in $M_{n}(\mathfrak{F})$ is an additive commutator $[A, B]=$ $A B-B A$. This supplemented an earlier result by Shoda [177] for fields of characteristic 0 .

In [77] Albert proved that a finite dimensional ordered division algebra is necessarily commutative. This does not hold for infinite dimensional algebras, for Hilbert has given an example in the second edition of his Grundlagen der Geometrie of a "twisted" power series division ring which is not commutative and which can be ordered. It is interesting to note that Hilbert's first attempt to give such an example in the first edition of Grundlagen can be seen to be wrong by invoking Albert's theorem!

Another pretty result of Albert's gives a determination of the finite dimensional absolute valued algebras over $\boldsymbol{R}$. By this we mean a (nonassociative) algebra over $\boldsymbol{R}$ which has a map $a \rightarrow|a|$ into $\boldsymbol{R}$ with the usual 
properties:

(1) $|a| \geqq 0$ and $|a|=0$ if and only if $a=0$;

(2) $|a+b| \leqq|a|+|b|$;

(3) $|\alpha a|=|\alpha||a|$ for $\alpha \in \boldsymbol{R}$;

(4) $|a b|=|a||b|$.

It had been conjectured by Kaplansky that if such an algebra has a unit, then it is alternative, and hence, by a classical result, it is necessarily either $\boldsymbol{R}, \boldsymbol{C}$, Hamilton's quaternion algebra $\boldsymbol{H}$, or Cayley's octonions $\boldsymbol{O}$. Moreover, in all cases $|a|=|a \bar{a}|^{1 / 2}$, where $\bar{a}$ is the usual conjugate. Albert proved this [96] and also showed that if all the conditions except the existence of a unit hold, then the algebra is an isotope of $\boldsymbol{R}, \boldsymbol{C}, \boldsymbol{H}$ or $\boldsymbol{O}$. This result was extended [101] to algebraic algebras over $\boldsymbol{R}$ not assumed to be finite dimensional.

Albert's last published paper [149]—published posthumously_proves an interesting theorem on quaternion algebras: If $\mathfrak{A}_{1}$ and $\mathfrak{A}_{2}$ are two (generalized) quaternion division algebras over a field $\mathfrak{F}$ and $\mathfrak{A}_{1} \otimes_{\mathfrak{F}} \mathfrak{A}_{2}$ is not a division algebra, then $\mathfrak{A}_{1}$ and $\mathfrak{A}_{2}$ have a common quadratic subfield.

Our recital of Albert's major achievements gives no indication of his methods or, more broadly speaking, of his mathematical style, which was highly individualistic. Perhaps its most characteristic qualities were the directness of his approach to a problem and his power and stamina to stick with it until he achieved a complete solution. He had a fantastic insight into what might be accomplished by intricate and subtle calculations of a highly original character. At times he could have obtained simpler proofs by using more sophisticated tools (e.g. representation theory), and one can almost always improve upon his arguments. However, this is of secondary importance compared to the first breakthrough which establishes a definitive result. It was in this that Albert really excelled. He regarded himself as a "pure" algebraist and in a sense he was. However, his best work - the solution of the problem of multiplication algebras of Riemann matrices-had its origin in another branch of mathematics. Moreover, he could exploit analytic and number theoretic results when he needed them-as he did in this instance.

\section{Bibliography of A. A. Albert}

\section{Books}

1. Modern Higher Algebra, Univ. of Chicago Press, Chicago, Ill., 1937.

2. Structure of Algebras, Amer. Math. Soc. Colloq. Publ., vol. 24, Amer. Math. Soc. Providence, R.I., 1939. MR 1, 99.

3. Introduction to Algebraic Theories, Univ. of Chicago Press, Chicago, Ill., 1941. MR 2, 241. 
4. College Algebra, McGraw-Hill, New York, 1946; reprinted by Univ. of Chicago Press, Chicago, Ill., 1963.

5. Solid Analytic Geometry, McGraw-Hill, New York, 1949.

6. Fundamental concepts of higher algebra, Univ. of Chicago Press, Chicago, Ill., 1958. MR 20 \#5190.

7. (with Reuben Sandler), An Introduction to Finite Projective Planes, Holt, Rinehart and Winston, New York, 1968. MR 37 \#3435.

8. (edited by A. A. Albert), Studies in Modern Algebra, Studies in Math., vol. 2, Math. Assoc. of Amer., distributed by Prentice-Hall, Englewood Cliffs, N.J., 1963. MR 26 \#3750.

\section{Articles}

9. A determination of all associative algebras in two, three and four units over a nonmodular field $\mathfrak{F}$, M.S. Thesis, University of Chicago, Chicago, Ill., 1927.

10. Algebras and their radicals, and division algebras, $\mathrm{Ph} . \mathrm{D}$. Dissertation, University of Chicago, Chicago, Ill., 1928.

11. A determination of all normal division algebras in sixteen units, Trans. Amer. Math. Soc. 31 (1929), 253-260.

12. On the rank equation of any normal division algebra, Bull. Amer. Math. Soc. 35 (1929), 335-338.

13. The rank function of any simple algebra, Proc. Nat. Acad. Sci. U.S.A. 15 (1929), 372-376.

14. On the structure of normal division algebras, Ann. of Math. 30 (1929), 322-338.

15. Normal division algebras in $4 p^{2}$ units, $p$ an odd prime, Ann. of Math. 30 (1929), 583-590.

16. The structure of an algebra which is a direct product of rational generalized quaternion division algebras, Ann. of Math. 30 (1929), 621-625.

17. On the structure of pure Riemann matrices with non-commutative multiplication algebras, Proc. Nat. Acad. Sci. U.S.A. 16 (1930), 308-312.

18. On direct products, cyclic division algebras, and pure Riemann matrices, Proc. Nat. Acad. Sci. U.S.A. 16 (1930), 313-315.

19. The non-existence of pure Riemann matrices with normal multiplication algebras of order sixteen, Ann. of Math. 31 (1930), 375-380.

20. A necessary and sufficient condition for the non-equivalence of any two rational generalized quaternion division algebras, Bull. Amer. Math. Soc. 36 (1930), 535-540.

21. Determinations of all normal division algebras in thirty-six units of type $R_{2}$, Amer. J. Math. 52 (1930), 283-292.

22. A note on an important theorem on normal division algebras, Bull. Amer. Math. Soc. 36 (1930), 649-650.

23. New results in the theory of normal division algebras, Trans. Amer. Math. Soc. 32 (1930), 171-195.

24. The integers of normal quartic fields, Ann. of Math. 31 (1930), 381-418.

25. A determination of the integers of all cubic fields, Ann. of Math.31 (1930), 550-566.

26. A construction of all non-commutative rational division algebras of order eight, Ann. of Math. 31 (1930), 567-576.

27. Normal division algebras of order $2^{2 m}$, Proc. Nat. Acad. Sci. U.S.A. 17 (1931), 389-392.

28. The structure of pure Riemann matrices with noncommutative multiplication algebras, Palermo Rendiconti 55 (1931), 57-115.

29. On direct products, cyclic division algebras and pure Riemann matrices, Trans. Amer. Math. Soc. 33 (1931), 219-234; Correction, 999. 
30. On normal division algebras of type $R$ in thirty-six units, Trans. Amer. Math. Soc. 33 (1931), 235-243.

31. On the Wedderburn norm condition for cyclic algebras, Bull. Amer. Math. Soc. 37 (1931), 301-312.

32. A note on cyclic algebras of order 16, Bull. Amer. Math. Soc. 37 (1931), 727-730.

33. On direct products, Trans. Amer. Math. Soc. 33 (1931), 690-711.

34. Division algebras over an algebraic field, Bull. Amer. Math. Soc. 37 (1931), $777-784$.

35. The structure of matrices with any normal division algebra of multiplications, Ann. of Math. 32 (1931), 131-148.

36. On the construction of cyclic algebras with a given exponent, Amer. J. Math. 54 (1932), 1-13.

37. Algebras of degree $2^{e}$ and pure Riemann matrices, Ann. of Math.33 (1932), 311-318.

38. A construction of non-cyclic normal division algebras, Bull. Amer. Math. Soc. 38 (1932), 449-456.

39. Normal division algebras of degree four over an algebraic field, Trans. Amer. Math. Soc. 34 (1932), 363-372.

40. On normal simple algebras, Trans. Amer. Math. Soc. 34 (1932), 620-625.

41. A note on division algebras of order sixteen, Bull. Amer. Math. Soc. 38 (1932), 703-706.

42. (with H. Hasse), A determination of all normal division algebras over an algebraic number field, Trans. Amer. Math. Soc. 34 (1932), 722-726.

43. A note on the equivalence of algebras of degree two, Bull. Amer. Math. Soc. 39 (1933), 257-258.

44. On primary normal division algebras of degree eight, Bull. Amer. Math. Soc. 39 (1933), 265-272.

45. Non-cyclic algebras of degree and exponent four, Trans. Amer. Math. Soc. 35 (1933), 112-121.

46. The integers represented by sets of ternary quadratic forms, Amer. J. Math. 55 (1933), 274-292.

47. On universal sets of positive ternary quadratic forms, Ann. of Math. 34 (1933), 875-878.

48. A note on the Dickson theorem on universal ternaries, Bull. Amer. Math. Soc. 39 (1933), 585-588.

49. Normal division algebras over algebraic number fields not of finite degree, Bull. Amer. Math. Soc. 39 (1933), 746-749.

50. Cyclic fields of degree eight, Trans. Amer. Math. Soc. 35 (1933), 949-964.

51. On the construction of Riemann matrices. I, Ann. of Math. 35 (1934), 1-28.

52. On a certain algebra of quantum mechanics, Ann. of Math. 35 (1934), 65-73.

53. Normal division algebras of degree 4 over $F$ of characteristic 2, Amer. J. Math. 56 (1934), 75-86.

54. Integral domains of rational generalized quaternion algebras, Bull. Amer. Soc. 40 (1934), 164-176.

55. On certain imprimitive fields of degree $p^{2}$ over $P$ of characteristic $p$, Ann. of Math. 35 (1934), 211-219.

56. Normal division algebras over a modular field, Trans. Amer. Math. Soc. 36 (1934), 388-394.

57. A solution of the principal problem in the theory of Riemann matrices, Ann. of Math. 35 (1934), 500-515.

58. Cyclic fields of degree $p^{r}$ over $F$ of characteristic $p$, Bull. Amer. Math. Soc. 40 (1934), 625-631. 
59. On normal Kummer fields over a non-modular field, Trans. Amer. Math. Soc. 36 (1934), 885-892.

60. Involutorial simple algebras and real Riemann matrices, Proc. Nat. Acad. Sci. U.S.A. 20 (1934), 676-681.

61. The principal matrices of a Riemann matrix, Bull. Amer. Math. Soc. 40 (1934), 843-846.

62. A note on the Poincaré theorem on impure Riemann matrices, Ann. of Math. 36 (1935), 151-156.

63. On the construction of Riemann matrices. II, Ann. of Math. 36 (1935), 376-394.

64. On cyclic fields, Trans. Amer. Math. Soc. 37 (1936), 454-462.

65. Involutorial simple algebras and real Riemann matrices, Ann. of Math. 36 (1935), 886-894.

66. Normal division algebras of degree $p^{e}$ over $P$ of characteristic $p$, Trans. Amer. Math. Soc. 39 (1936), 183-188.

67. Simple algebras of degree $p^{e}$ over a centrum of characteristic $p$, Trans. Amer. Math. Soc. 40 (1936), 112-126.

68. A note on matrices defining total real fields, Bull. Amer. Math. Soc. 43 (1937), 242-244.

69. p-algebras over a field generated by one indeterminate, Bull. Amer. Math. Soc. 43 (1937), 733-736.

70. Normalized integral bases of algebraic number fields. I, Ann. of Math. 38 (1937), 923-957.

71. A quadratic form problem in the calculus of variations, Bull. Amer. Math. Soc. 44 (1938), 250-253.

72. Non-cyclic algebras with pure maximal subfields, Bull. Amer. Math Soc. 44 (1938), 576-579.

73. Symmetric and alternate matrices in an arbitrary field. I, Trans. Amer. Math. Soc. 43 (1938), 386-436.

74. Quadratic null forms over a function field, Ann. of Math. 39 (1938), 494-505.

75. On cyclic algebras, Ann. of Math. 39 (1938), 669-682.

76. A note on normal division algebras of prime degree, Bull. Amer. Math. Soc. 44 (1938), 649-652.

77. On ordered algebras, Bull. Amer. Math. Soc. 46 (1940), 521-522. MR 1, 328.

78. On p-adic fields and rational division algebras, Ann. of Math. (2) 41 (1940), 674-693. MR 2, 123.

79. A rule for computing the inverse of a matrix, Amer. Math. Monthly 48 (1941), 198-199. MR 2, 243. 265 .

80. Division algebras over a function field, Duke Math. J. 8 (1941), 750-762. MR 3,

81. Quadratic forms permitting composition, Ann. of Math. (2) 43 (1942), 161-177. MR 3, 261.

82. Non-associative algebras. I. Fundamental concepts and isotopy, Ann. of Math.

(2) 43 (1942), 685-707. MR 4, 186.

83. Non-associative algebras. II. New simple algebras, Ann. of Math. (2) 43 (1942), 708-723. MR 4, 186.

84. The radical of a non-associative algebra, Bull. Amer. Math. Soc. 48 (1942), 891-897. MR 4, 130.

85. An inductive proof of Descartes' rule of signs, Amer. Math. Monthly 50 (1943), 178-180. MR 4, 195.

86. A suggestion for a simplified trigonometry, Amer. Math. Monthly 50 (1943), 251-253. 
87. Quasigroups. I, Trans. Amer. Math. Soc. 54 (1943), 507-519. MR 5, 229.

88. Algebras derived by non-associative matrix multiplication, Amer. J. Math. 66 1944), 30-40. MR 5, 227.

89. The matrices of factor analysis, Proc. Nat. Acad. Sci. U.S.A. 30 (1944), 90-95 MR 5, 209.

90. The minimum rank of a correlation matrix, Proc. Nat. Acad. Sci. U.S.A. 30 (1944), 144-146. MR 6, 6.

91. Quasigroups. II, Trans. Amer. Math. Soc. 55 (1944), 401-419. MR 6, 42.

92. Two element generation of a separable algebra, Bull. Amer. Math. Soc. 50 (1944), 786-788. MR 6, 115.

93. Quasiquaternion algebras, Ann. of Math. (2) 45 (1944), 623-638. MR 6, 115.

94. On Jordan algebras of linear transformations, Trans. Amer. Math. Soc. 59 (1946), 524-555. MR 8, 63.

95. The Wedderburn principal theorem for Jordan algebras, Ann. of Math. (2) 48 (1947), 1-7. MR 8, 435 .

96. Absolute valued real algebras, Ann. of Math. (2) 48 (1947), 495-501; Correction in Bull. Amer. Math. Soc. 55 (1949), 1191. MR 8, 561; 11, 76.

97. A structure theory for Jordan algebras, Ann of Math (2) 48 (1947), 546-567. MR 9, 77.

98. On the power-associativity of rings, Summa Brasil. Math. 2 (1948), no. 2, 21-33. MR 10, 97. 349 .

100. On right alternative algebras, Ann. of Math. (2) 50 (1949), 318-328. MR 10, 503.

101. Absolute-valued algebraic algebras, Bull. Amer. Math. Soc. 55 (1949), 763768. MR 11, 76.

102. A theory of trace-admissible algebras, Proc. Nat. Acad. Sci. U.S.A. 35 (1949), 317-322. MR 11, 6.

103. Almost alternative algebras, Portugal. Math. 8 (1949), 23-36. MR 11, 316.

104. A note on the exceptional Jordan algebra, Proc. Nat. Acad. Sci. U.S.A. 36 (1950), 372-374. MR 12, 5 .

105. A theory of power-associative commutative algebras, Trans. Amer. Math. Soc. 69 (1950), 503-527. MR 12, 475.

106. New simple power-associative algebras, Summa Brasil. Math. 2 (1951), 183194. MR 14, 11 .

107 Power-associative algebras, Proc. Internat. Congress Math. (Cambridge, Mass., 1950), vol. 2, Amer. Math. Soc., Providence, R.I., 1952, pp. 25-32. MR 13, 527.

108. On nonassociative division algebras, Trans. Amer. Math. Soc. 72 (1952), 296309. MR 13, 816.

109. On simple alternative rings, Canad. J. Math. 4 (1952), 129-135. MR 14, 11.

110. On commutative power-associative algebras of degree two, Trans. Amer. Math. Soc. 74 (1953), 323-343. MR 14, 614.

111. Rational normal matrices satisfying the incidence equation, Proc. Amer. Math. Soc. 4 (1953), 554-559. MR 15, 94.

112. The structure of right alternative algebras, Ann. of Math. (2) 59 (1954), 408-417. MR 15, 774.

113. (with M. S. Frank), Simple Lie algebras of characteristic p, Univ. e Politec. Torino. Rend. Sem. Mat. 14 (1954/55), 117-139. MR 18, 52.

114. Leonard Eugene Dickson, 1874-1954, Bull. Amer. Math. Soc. 61 (1955), 331345. MR 17, 2. 
115. On involutorial algebras, Proc. Nat. Acad. Sci. U.S.A. 41 (1955), 480-482. MR 17, 9.

116. On Hermitian operators over the Cayley algebra, Proc. Nat. Acad. Sci. U.S.A. 41 (1955), 639-640. MR 17, 123.

117. A property of special Jordan algebras, Proc. Nat. Acad. Sci. U.S.A. 42 (1956), 624-625. MR 18, 375.

118. The norm form of a rational division algebra, Proc. Nat. Acad. Sci. U.S.A. 43 (1957), 506-509. MR 19, 246.

119. On certain trinomial equations in finite fields, Ann. of Math. (2) 66 (1957), 170178. MR 19, 394.

120. (with B. Muckenhoupt), On matrices of trace zero, Michigan Math. J. 4 (1957), 1-3. MR 18, 786.

121. On partially stable algebras, Trans. Amer. Math. Soc. 84 (1957), 430-443. MR 19, 1156.

122. (with N. Jacobson), On reduced exceptional simple Jordan algebras, Ann. of Math. (2) 66 (1957), 400-417. MR 19, 527.

123. A property of ordered rings, Proc. Amer. Math. Soc. 8 (1957), 128-129. MR 18, 557.

124. (with John Thompson), Two element generation of the projective unimodular group, Bull. Amer. Math. Soc. 64 (1958), 92-93. MR 20 \#3211.

125. Addendum to the paper on partially stable algebras, Trans. Amer. Math. Soc. 87 (1958), 57-62. MR 19, 1157.

126. A construction of exceptional Jordan division algebras, Ann. of Math. (2) 67 (1958), 1-28. MR 19, 1036.

127. On the orthogonal equivalence of sets of real symmetric matrices, J. Math. Mech. 7 (1958), 219-235. MR 19, 1153.

128. A solvable exceptional Jordan algebra, J. Math. Mech. 8 (1959), 331-337. MR 21 \#681.

129. (with L. J. Paige), On a homomorphism property of certain Jordan algebras, Trans. Amer. Math. Soc. 93 (1959), 20-29. MR 21 \#7240.

130. (with John Thompson), Two-element generation of the projective unimodular group, Illinois J. Math. 3 (1959), 421-439. MR 21 \#5681.

131. Finite noncommutative division algebras, Proc. Amer. Math. Soc. 9 (1958), 928-932. MR 21 \#1994.

132. Finite division algebras and finite planes, Proc. Sympos. Appl. Math., Vol. 10, Amer. Math. Soc., Providence, R.I., 1960, pp. 53-70. MR 22 \#6831.

133. On the collineation groups associated with twisted fields, Calcutta Math. Soc. Golden Jubilee Commemoration Volume (1958/59), part II, Calcutta Math. Soc., Calcutta, 1963, pp. 485-497. MR 28 \#3070.

134. On the collineation groups of certain non-desarguesian planes, Portugal. Math. 18 (1959), 207-224. MR 24 \#A473.

135. Generalized twisted fields, Pacific J. Math. 11 (1961), 1-8. MR 23 \#A182.

136. Isotopy for generalized twisted fields, An. Acad. Brazil. Ci. 33 (1961), 265-275. MR 25 \#3070.

137. On involutorial associative division algebras, Scripta Math. 26 (1963), 309-316. MR 31 \#3451.

138. On the nuclei of a simple Jordan algebra, Proc. Nat. Acad. Sci. U.S.A. 50 (1963), 446-447. MR 27 \#3679.

139. Finite planes for the high school, The Math. Teacher 55 (1962), 165-169.

140. A normal form for Riemann matrices, Canad. J. Math. 17 (1965), 1025-1029. MR 32 \#111. 
141. On exceptional Jordan division algebras, Pacific J. Math. 15 (1965), 377-404. MR 32 \#130.

142. On associative division algebras of prime degree, Proc. Amer. Math. Soc. 16 (1965), 799-802. MR 31 \#3452.

143. The finite planes of Ostrom, Bol. Soc. Mat. Mexicana (2) 11 (1966), 1-13. MR 37 \#6826.

144. New results on associative division algebras, J. Algebra 5 (1967), 110-132. MR 34 \#2617.

145. On some properties of biabelian fields, An. Acad. Brasil. Ci. 38 (1966), 217-221. MR 34 \#7498.

146. On certain polynomial systems, Scripta Math. 28 (1967), 15-19. MR 35 \#2865.

147. On associative division algebras (Retiring Presidential Address), Bull. Amer. Math. Soc. 74 (1968), 438-454. MR 36 \#5166.

148. A note on certain cyclic algebras, J. Algebra 14 (1970), 70-72. MR 40 \#4297.

149. Tensor products of quaternion algebras, Proc. Amer. Math. Soc. 35 (1972), 65-66. MR 45 \#6855.

\section{REFERENCES}

150. S. A. Amitsur, On central division algebras, Israel J. Math. 12 (1972), 408-420. MR 47 \#6763.

151. R. Block, New simple Lie algebras of prime characteristic, Trans. Amer. Math. Soc. 89 (1958), 421-449. MR 20 \#6446.

152. R. Brauer, Über Systeme hyperkomplexer Zahlen, Math. Z. 29 (1929), 79-107. 153. — Untersuchungen über die arithmetischen Eigenschaften von Gruppen linearer Substitutionen. II, Math. Z. 31 (1930), 733-747.

154. - On normal division algebras of index five, Proc. Nat. Acad. Sci. U.S.A. 24 (1938), 243-246.

155. R. Brauer, H. Hasse and E. Noether, Beweis eines Hauptsatzes in der Theorie der Algebren, J. Reine Angew. Math. 167 (1931), 399-404.

156. F. Cecioni, Sopra un tipo di algebre prive di divisore dello zero, Rend. Circ. Mat. Palermo 47 (1923), 209-254.

157. P. M. Cohn, On homomorphic images of special Jordan algebras, Canad. J. Math. 6 (1954), 253-264. MR 15, 678.

158. L. E. Dickson, New division algebras, Trans. Amer. Math. Soc. 28 (1926), 207234.

159. - Construction of division algebras, Trans. Amer. Math. Soc. 32 (1930), 319-334.

160. M. S. Frank, A new class of simple Lie algebras, Proc. Nat. Acad. Sci. U.S.A. 40 (1954), 713-719. MR 16, 562.

161. H. Hasse, Zum Existenzsatz von Grunwald in der Klassenkörpertheorie, J. Reine Angew. Math. 188 (1950), 40-64. MR 12, 677.

162. G. P. Hochschild, Semi-simple algebras and generalized derivations, Amer. J. Math. 64 (1942), 677-694. MR 4, 71.

163. F. D. Jacobson and N. Jacobson, Classification and representation of semisimple Jordan algebras, Trans. Amer. Math. Soc. 65 (1949), 141-169. MR 10, 588.

164. N. Jacobson, A note on nonassociative algebras, Duke Math. J. 3 (1937), 544-548.

165. - A theorem on the structure of Jordan algebras, Proc. Nat. Acad. Sci. U.S.A. 42 (1956), 140-147. MR 17, 822.

166. — Structure and representations of Jordan algebras, Amer. Math. Soc. Colloq. Publ., vol. 39, Amer. Math. Soc., Providence, R.I., 1968. MR 40 \#4330. 
167. P. Jordan, Über eine Klasse nichtassoziativer hyperkomplexer Algebren, Nachr. Ges. Wiss. Göttingen 1932, 569-575.

168. P. Jordan, J. von Neumann and E. P. Wigner, On an algebraic generalization of the quantum mechanical formalism, Ann. of Math. 36 (1934), 29-64.

169. G. K. Kalisch, On special Jordan algebras, Trans. Amer. Math. Soc. 61 (1947), 482-494. MR 8, 561.

170. E. Kleinfeld, Simple alternative rings, Ann. of Math. (2) 58 (1953), 544-547. MR 15, 392.

171. — Alternative nil rings, Ann. of Math. (2) 66 (1957), 395-399. MR 19, 383.

172. A. I. Kostrikin and I. R. Šafarevič, Graded Lie algebras of finite characteristic, Izv. Akad. Nauk SSSR Ser. Mat. 33 (1969), 251-322=Math. USSR Izv. 3 (1969), 237-304. MR 40 \#5680.

173. K. McCrimmon, Finite power associative division rings, Proc. Amer. Math. Soc. 17 (1966), 1173-1177. MR 34 \#4319.

174. — A note on finite division rings, Proc. Amer. Math. Soc. 23 (1969), 598-600. MR 40 \#7320.

175. M. L. Racine, A note on quadratic Jordan algebras of degree 3, Trans. Amer. Math. Soc. 164 (1972), 93-103. MR 46 \#3582.

176. C. Rosati, Sulle matrici di Riemann, Rend. Circ. Mat. Palermo 53 (1929), 79-134.

177. K. Soda, Einige Sätze über Matrizen, Japan J. Math. 13 (1936), 361-365.

178. C. L. Siegel, Lectures on Riemann matrices, Tata Institute of Fundamental Research Lectures on Math., no. 28, Tata Institute of Fundamental Research, Bombay, 1963. MR 42 \#1861.

179. T. A. Springer, The classification of reduced exceptional simple Jordan algebras, Nederl. Akad. Wetench. Proc. Ser. A 63=Indag. Math. 22 (1960), 414-422. MR 26 \#5035.

180. O. Teichmüller, Multiplikation zyklischer Normalringen, Deutsch. Math. 1936, 197-238.

181. — - p-Algebren, Deutsch. Math. 1936, 362-388.

182. S. Wang, A counter-example to Grunwald's theorem, Ann. of Math. (2) 49 (1948), 1008-1009. MR 10, 231.

183. —- On Grunwald's theorem, Ann. of Math. (2) 51 (1950), 471-484. MR 11, 489.

184. J. H. M. Wedderburn, A type of primitive algebra, Trans. Amer. Math. Soc. 15 (1914), 162-166.

185. - On division algebras, Trans. Amer. Math. Soc. 22 (1921), 129-135.

186. H. Weyl, On generalized Riemann matrices, Ann. of Math. 35 (1934), 714-729.

187. —_ Generalized Riemann matrices and factor sets, Ann. of Math. 37 (1936), 709-745.

188. D. Zelinsky, A. A. Albert, Amer. Math. Monthly 80 (1973), 661-665. MR 47 \#1572.

Department of Mathematics, Yale University, New Haven, Connecticut 06520 